\title{
Augmentation of Candida albicans adhesion to denture materials influenced by surface topography and tobacco components
}

\begin{abstract}
The aim of this study was to investigate the influence of tobacco components, tobacco extract (TE) nicotine and Cotinine on fungal colonization and biofilm formation on two acrylic denture resins. Surface topography of the denture materials and alteration of fungal susceptibility to an anti-fungal agent, Nystatin of both planktonic and biofilm fungal cells grown on the acrylic resins were investigated. Ivocap and Lucitone 199 polished and roughened acrylic resin discs were fabricated and randomly assigned to control, tobacco extract-, nicotine-, and Cotinine-treated groups. Candida albicans biofilm was prepared on the resin discs in the presence or absence of tobacco components. The relative number of viable fungal cells was determined by the MTT assay. Quantization of biofilm growth was performed by dry weight analysis. Nystatin susceptibility [minimum inhibitory concentration (MIC)] was determined by microdilution method. Statistical significance was evaluated using ANOVA followed by Fisher's test $(p<0.05)$. The study showed that the roughened discs had significantly greater numbers of C.albicans than the polished discs, Lucitone 199 acrylic discs promoted significantly more fungal biofilm growth than Ivocap discs, and the presence of tobacco components significantly enhanced biofilm formation on both types of acrylic resin. Dry weight analysis showed similar results. The Nystatin susceptibility assay showed that cells treated with tobacco components were more resistant to this antifungal agent.
\end{abstract}

Volume 9 Issue I - 2018

\author{
Jenny Phung Stevens,' Paivi Samant,' Vinay \\ Jain,' Jegdish $P$ Babu' \\ 'Department of Prosthodontics, The University of Tennessee \\ Health Science Center, USA \\ ${ }^{2}$ Department of Bio Science Research, The University of \\ Tennessee Health Science Center, USA
}

Correspondence: Jegdish P Babu, Professor, Department of Bio Science Research, College of Dentistry, The University of Tennessee Health Science Center, Memphis, TN 38I63, USA, Email jbabu@uthsc.edu

Received: December 29, 2018 | Published: January 31, 2018

Keywords: tobacco components, nicotine, cotinine, candida albicans, biofilm, ivocap, lucitone 199 , nystatin

\section{Introduction}

Denture stomatitis, a common oral disease, can induce pathologic changes to the denture-bearing tissues. These tissue changes can vary from localized hyperemia to diffused erythema to papillary hyperplasia. ${ }^{1,2}$ Patients with severe denture stomatitis may experience pain, itching, and/or burning sensation..$^{3-5}$ Studies have shown that the prevalence of denture stomatitis in the edentulous population ranges from $15 \%$ to over $70 \%$ with more cases found in the elderly, women, smokers, and immunocompromised..$^{6-12}$ Candida albicans adherence and colonization of denture prostheses has been shown to induce denture stomatitis. ${ }^{13,14}$ In general, C.albicans species exist as commensal organisms in about $33 \%$ of the adult dentate patients and about $75 \%$ of the complete denture patients. Smears obtained from the intaglio surface of dentures often demonstrate the presence of the invasive filamentous hyphal form. ${ }^{8-17}$ Denture base resins are susceptible to fungal colonization in the oral environment. Surface roughness is one of the factors that aids in the initial fungal attachment. Surface defects such as scratches, cracks, and porosities serve as protective surfaces for microorganisms to bind. ${ }^{18,19}$ One of the more common denture resins used today is polymethylmethacrylate (PMMA), a polymer developed in the $1930 \mathrm{~s} .{ }^{20}$ PMMA can be classified as heat-activated or chemically-activated resin based on the method of polymerization. Heat-activated PMMA can be processed by compression-molded, injection-molded, or microwave-processed techniques. ${ }^{21}$ Physical characteristics of PMMA are dependent on the type of resin as well as the processing technique. Manufacturers of the injection-molded resin claim that the "cross-linked, high impact thomopolymer" obtained after polymerization offers excellent polishability and helps reduce plaque build-up..$^{22}$ It has been reported that use of tobacco products induces fungal denture stomatitis in susceptible patients. ${ }^{23}$ According to a study by Arendorf et al. there are higher numbers of Candida in tobacco smokers as compared to non-smokers. ${ }^{24}$ Salivary nicotine and cotinine are widely used in clinical and epidemiological smoking studies. ${ }^{25}$ Nicotine is a weak base with a $\mathrm{pKa}$ value that approaches 8 . When salivary $\mathrm{pH}$ increases, the non-ionized form of nicotine is absorbed across the buccal and nasal membranes. ${ }^{26}$ Cotinine, the major metabolite of nicotine, has been reported to have a $\mathrm{pKa}$ of 5. ${ }^{27}$ The concentration of cotinine in saliva is variable depending on an individual's nicotine metabolism and the salivary $\mathrm{pH}$. Salivary concentrations of nicotine and cotinine can be affected by many factors such as cigarette brand, length of cigarette, gender, puffing behavior, and testing methods. ${ }^{28}$ Robson et al. ${ }^{29}$ reported that salivary nicotine concentrations ranged from $0.36 \mu \mathrm{g} / \mathrm{ml}$ to more than $4.6 \mu \mathrm{g} /$ $\mathrm{ml}$ and cotinine concentrations ranged from $0.0096 \mu \mathrm{g} / \mathrm{ml}$ to more than $1.4 \mu \mathrm{g} / \mathrm{ml}$ in individuals who smoked 2-20 cigarettes per day. In contrast, Cote et al. ${ }^{28}$ reported each cigarette's nicotine concentration to be between $100 \mu \mathrm{g} /$ cigarette to more than $2650 \mu \mathrm{g} /$ cigarette. While smoking has been implicated as one of the factors contributing to denture stomatitis; however, its exact pathogenic influence has yet to be demonstrated. 
Nystatin, discovered and isolated in the early 1950s from Streptomyces noursei, ${ }^{30}$ is one of the most common antifungals used to treat $C$. albicans-induced denture stomatitis. It is a broad spectrum polygene agent shown to inhibit Candida cell growth and division. ${ }^{31}$ It is available in oral rinses, creams, and slow-releasing tablets. $^{32}$ Studies have shown that prophylactic oral application of Nystatin may prevent systemic spread of oral candidal infection in immunocompromised individuals ${ }^{33}$ and suppresses candidal adhesion to denture acrylic surfaces..$^{34,35}$ Understanding the underlying factors leading to fungal adhesion to denture acrylic resin surfaces and the roles that nicotine and cotinine play in biofilm formation may help alleviate the problems associated with denture-induced stomatitis. This first aim of this study was to evaluate the effect that tobacco components had on two different denture base acrylic resins with polished and roughened surface topography. The second aim was to test Nystatin susceptibility of Candida grown in the presence of tobacco components. The null hypothesis was that the surface topography, type of acrylic resin, and tobacco treatment would not affect fungal growth.

\section{Materials and methods}

\section{Preparation of experimental denture discs}

Acrylic resin discs $(10 \mathrm{mmx} 1 \mathrm{~mm})$ were prepared from polymethyl methacrylate (PMMA) obtained from two different manufacturers. $10 \mathrm{~mm}$ diameter vinyl polysiloxane (VPS) (Dentsply International Inc., York, PA) rods were prepared and invested in type III dental stone (Whip Mix Corp., Louisville, KY) in the respective flasks. Ivocap (Ivoclar Vivodent AG, Schaan, Liechtenstein) was processed via the injection-molded technique using $5.0 \mathrm{ml}$ diameter wax sprues and then boiled. Then $20 \mathrm{~g}$ polymer and $30 \mathrm{ml}$ monomer were mixed together for 5 minutes in the Cap Vibrator and polymerized for 35 min, the experimental materials were cooled. The resin rods were cut into sample discs $(10 \mathrm{mmx} 1 \mathrm{~mm})$ using an electric saw (Cut-Off Saw, Harbor Freight Tools, Calabasas, CA). A roughened surface was obtained by using an acrylic bur (Komet, Rock Hill, SC) to abrade the discs. To obtain a polished surface, acrylic discs were pumiced using a combination of medium and fine pumice (Whip Mix Corp.) and polished using polishing compound (Grobet, Carlstadt, NJ). Lucitone 199 (Dentsply International Inc., York, NY, USA) was mixed at 3.2:1 powder to liquid ratio. The VPS rods were removed after the stone was set. The acrylic resin was trial packed twice and the excess was removed before final flask closure. Lucitone 199 was polymerized using the long curing cycle. The same methods detailed above were used to obtain polished and roughened discs. The discs were stored in purified water for seven days to remove any residual monomer. They were decontaminated by rinsing with bleach, then soaking in $70 \%$ ethanol for 2 hours, and finally were exposed to UV light overnight. The discs were routinely tested for sterility by culturing overnight in tissue culture media.

\section{Tobacco components tested}

Nicotine and cotinine were obtained from Sigma Chemical Co (St. Louis, MO, USA). Tobacco extracts were prepared by immersing the cigar tobacco leaves $(10 \mathrm{~g})$ in paraffin-stimulated clarified whole human saliva for 4 hours and then the mixture was subjected to sonication and the mixture was left on a stirrer at $4 \mathrm{C}$ for 6 hours. The supernatant was collected by centrifugation and then freeze-dried.
The tobacco extract and its products were suspended in phosphate buffered saline (PBS) to a concentration of $10.0 \mu \mathrm{g} / \mathrm{ml}$ for the study.

\section{Culturing of fungal cells and assay for their adhesion to denture discs}

Candida albicans 18807 was obtained from the American Type Culture Collection (ATCC, Manassas, VA) was grown in Sabouraud's broth (SAB, Gibco, and Grand Island, NY) at $30^{\circ} \mathrm{C}$ for 48 hours. The cells were washed with PBS and suspended to contain 1x107 cells $/ \mathrm{ml}$ using a spectrophotometer. Triplicate samples of each test disc were incubated with $1.0 \mathrm{ml}$ of fungal cells along with $100 \mu \mathrm{l}$ of tobacco products for $24 \mathrm{~h}$. The bound cells were collected from each disc. The biofilm-grown cells were prepared by placing two $\mathrm{ml}$ of fungal suspension in a sterile 24-well multi well culture plate (Becton Dickonson and Co., Franklin Lakes, NJ), with appropriate test disc (in triplicate) along with $100 \mu \mathrm{l}$ of tobacco products and incubated at $30^{\circ} \mathrm{C}$ for 7 days. During this period, an aliquot $(0.1 \mathrm{ml})$ of SAB supplemented with $10 \%$ sucrose was added each day. Discs incubated with $\mathrm{SAB}$ alone served as controls. Biofilm-grown cells from each disc were collected.

\section{Assessment of fungal adherence to the denture discs}

The number of freshly cultured and biofilm-grown fungal cells adhered to the denture discs in the presence or absence of tobacco components was determined by MTT cell viability assay. In brief, $50 \mu 1$ of MTT [3-(4,5-dimethylthiazol-2-yl)-2,5-diphenyltetrazolium bromide] solution ( $1 \mathrm{mg} / \mathrm{ml}$ medium; Sigma chemical Co.) was added to each well and incubated at $37^{\circ} \mathrm{C}$ for 2 hours. Then $0.1 \mathrm{ml}$ of solubilization solution (20\% (w/v) SDS, $2.5 \%(\mathrm{v} / \mathrm{v}) 1 \mathrm{~N} \mathrm{HCl}$ and $2.5 \%$ $(\mathrm{v} / \mathrm{v})$ acetic acid (80\%), $\mathrm{pH} 2)$ was added and incubated overnight. Production of formazan by viable cells was assessed by measuring the absorbance at a wavelength of $570 \mathrm{~nm}$ using an ELISA reader (Spectro star Nano, BMG Labtech, Inc., Ortenberg, Germany). All test samples (in triplicate) were assayed three times using the same protocol.

\section{Dry weight analysis and assessment nicotine effect on $C$ albicans sensitivity to Nystatin}

Equal numbers of Ivocap and Lucitone 199 sample discs were divided into polished and roughened groups. The polished and roughened groups were further subdivided into control and nicotinetreated samples. The eight groups had a total of 24 samples. The acrylic resin discs were pre-weighed prior to biofilm formation and the measurements were recorded. Biofilm of $C$. albicans was grown as described above in the presence of nicotine $(0.1 \mathrm{ml}$ of $10 \mu \mathrm{g} / \mathrm{ml}$ stock) or with PBS (control group). The susceptibility of planktonic and biofilm-grown $C$. albicans to ten different concentrations of the antifungal agent nystatin was determined using the National Committee for Clinical Laboratory Standards guidelines. Analytical grade nystatin powder (Sigma-Aldrich Co.) was dissolved in $100 \%$ dimethyl sulfoxide (DMSO) and absolute ethanol (3:2 ratio). The inoculum was prepared from one week old biofilm cells (105 CFU/ $\mathrm{ml})$. One $\mathrm{ml}$ aliquot of the suspension was placed in 10 different sterile glass tubes with varying concentrations of nystatin $(0.195 \mu \mathrm{g} /$ $\mathrm{ml}$ to $100 \mu \mathrm{g} / \mathrm{ml})$. The fungal cells were incubated for 24 hours. The minimum inhibitory concentration (MIC) of nystatin, determined visually, was defined as the lowest concentration at which there was 
$100 \%$ inhibition of C. albicans growth, as indicated by the absence of turbidity. Cells incubated with same solvent mixture without nystatin served as controls.

\section{Data analysis}

Results of MTT assays and dry weight analyses were evaluated by ANOVA followed by Fisher's test, with a statistical significance set at $p$ value $<0.05$.

\section{Results}

Differences in fungal colonization on denture discs influenced by surface topography and tobacco components were determined on freshly-cultured and biofilm-grown C. albicans by MTT viable cell assay. In this study we also examined the effect of extract prepared from tobacco leaves (TE), nicotine and Cotinine, the two major components of tobacco. Figure 1 demonstrates the differences in fungal adhesion to Ivocap denture discs. The tobacco extract (TE), nicotine and cotinine all enhanced the colonization of $C$. albicans both on polished and roughened denture discs, however, the roughened surfaces showed significantly $(P<0.026)$ more fungal cells adhered than the polished surfaces. On the roughened surfaces the number of viable fungal cells was significantly higher $(P<0.014)$ than the polished surfaces in the presence of nicotine and cotinine. TE had no significant difference in fungal adhesion between polished and roughened surfaces. The data of Figure 2 demonstrates the differences in fungal colonization on Lucitone denture discs. All three tobacco components had a significant $(P<0.011)$ effect on the number of viable fungal cells adhered to these discs compared to the control. No remarkable difference in fungal adhesion was seen when nicotine and cotinine was added to the polished denture discs compared to the TE. On the other hand, the number of viable $C$. albicans found on roughened surfaces was significantly higher $(\mathrm{P}<0.046)$ in the presence of both cotinine when compared to nicotine. Collectively these results suggest that the surface topography influences the fungal colonization, and the presence of tobacco components further exaggerates these differences. Table 1 demonstrates the average dry weight values of the eight sample groups. There were significantly greater number of cells found on the roughened surfaces than the polished surfaces of both types of resin $(p<0.039)$, more cells on Lucitone 199 than Ivocap discs $(p<0.041)$, and more cells in nicotine- and cotinine-treated groups than their respective control groups $(\mathrm{p}<0.024)$.

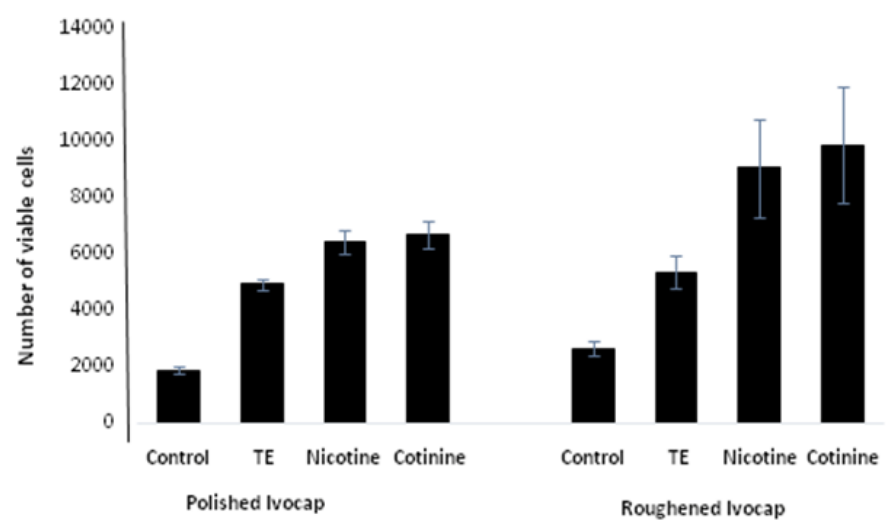

Figure I Comparison of adherent viable fungal cells influenced by surface topography of Ivocap acrylic discs and the effect of tobacco components.

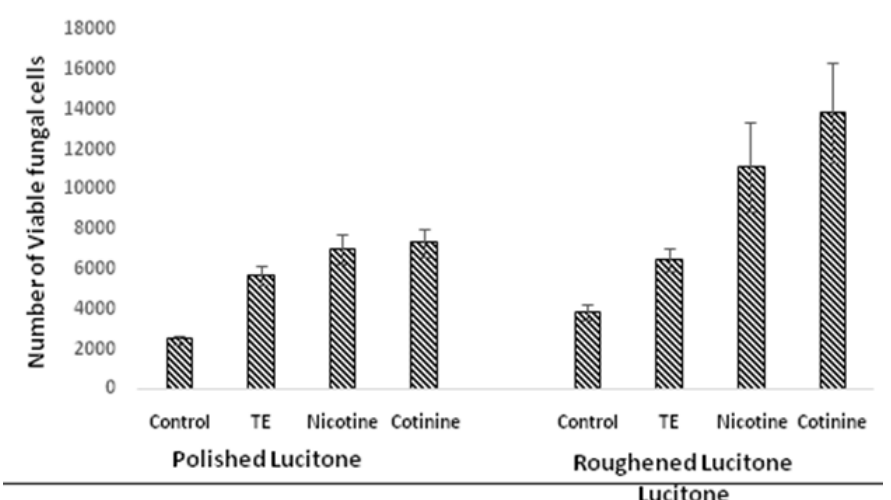

Figure 2 Demonstration of differences in C. albicans adherence to polished and roughened Lucitone 199 discs and the effect of tobacco products.

Table I Fungal biofilm load on denture acrylic material (micrograms \pm Standard error)

\begin{tabular}{lllll}
\hline Treated with & Ivocap & \multicolumn{3}{c}{ Lucitone } \\
\hline Buffer (control) & $1.33 \pm 0.29$ & $1.73 \pm 0.42$ & $1.87 \pm 0.42$ & $1.93 \pm 0.57$ \\
TE & $1.68 \pm 0.3 \mathrm{I}$ & $1.91 \pm 0.37$ & $2.08 \pm 0.53$ & $2.26 \pm 0.64$ \\
Nicotine & $1.99 \pm 0.39$ & $2.77 \pm 0.69$ & $3.5 \mathrm{I} \pm 0.77$ & $3.83 \pm 0.9 \mathrm{I}$ \\
Cotinine & $1.95 \pm 0.6 \mathrm{I}$ & $2.99 \pm 0.74$ & $3.92 \pm 0.84$ & $4.16 \pm 0.98$ \\
\hline
\end{tabular}

\section{Nystatin susceptibility analysis}

Figure 3 shows the minimum inhibitory concentration (MIC) of the nine tested groups. The nystatin MIC for cells in the planktonic (non-nicotine treated and non-denture disc) group was $1.56 \mu \mathrm{g} / \mathrm{ml}$. The nystatin MIC for the Ivocap polished and roughened, non-nicotine treated biofilm groups was $12.5 \mu \mathrm{g} / \mathrm{ml}$. The MIC for the Lucitone 199 polished and roughened, non-nicotine treated biofilm groups was $25 \mu \mathrm{g} / \mathrm{ml}$. The MIC of nicotine-treated biofilm groups was $50 \mu \mathrm{g} / \mathrm{ml}$.

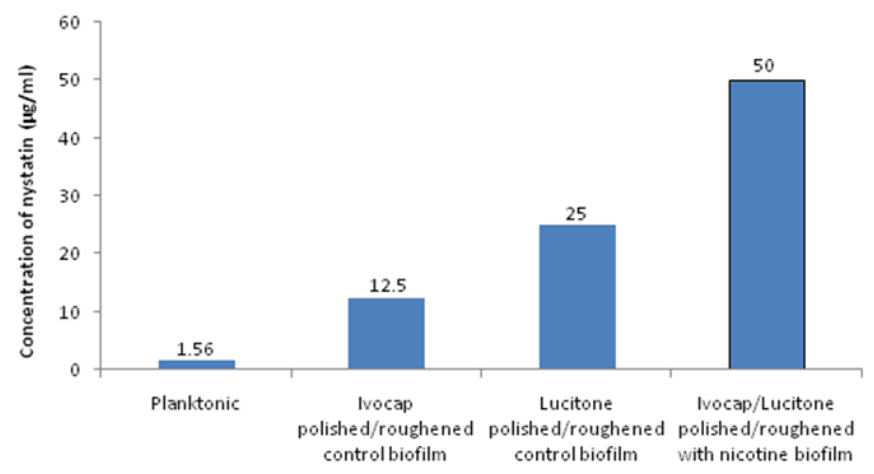

Figure 3 Differences in minimum inhibitory concentration of Nystatin on fungal biofilm formation influenced by surface topography and nicotine.

\section{Discussion}

The null hypothesis in this study was rejected since the surface irregularity, type of acrylic resin, and treatment with tobacco components affected fungal growth significantly. However, since MTT assay results varied among the different comparison groups, 
this suggested that the biofilm formation could have resulted from a complex phenomenon with a multifactorial origin. Given the vast range of denture base resins available, the results of this study could not be extrapolated and applied to other compression- and injection-molded materials. The results from the MTT assay of $C$. albicans grown on discs of different surface topography showed that some groups were affected by surface properties while others were not. For example, fungal growth on Ivocap nicotine- and cotininetreated roughened discs was significantly greater than on their polished counterparts. In contrast, rough surfaces on Ivocap and Lucitone 199 did not significantly enhance fungal growth when compared to the polished groups. These results were in agreement with other studies on surface topography which suggest that increased fungal growth on rough surfaces may be due to greater micro surface area available for attachment. Once attached, the fungal cells were less likely to be dislodged from these surfaces. ${ }^{18,19}$ In general, Lucitone 199 resin promoted more fungal growth than Ivocap. As evaluated by the MTT cell viability assay, Lucitone 199 polished and roughened denture discs treated with nicotine or cotinine promoted significantly more $C$. albicans growth than their Ivocap counterparts. However, in the absence of nicotine and cotinine, no significant differences were found between Lucitone 199 and Ivocap discs (polished or roughened). Fungal growth in nicotine- or cotinine- treated groups was significantly increased, except in the Ivocap polished groups, when compared to the control. This could be due to many factors that can prevent a study from reliably measuring nicotine and cotinine concentration intra orally as outlined by Cote et al. ${ }^{28}$ a pilot study was performed prior to the full study. The results suggested that a concentration of $1.0 \mu \mathrm{g} / \mathrm{ml}$ of nicotine and cotinine could induce fungal growth. This concentration was approximately the same concentrations reported by Robson et al. ${ }^{28}$ but lower than concentrations reported by Cote et al. ${ }^{28}$ since nicotine and cotinine elicited similar fungal responses, only nicotine was tested in the dry weight analysis. The results from this analysis showed significant differences in fungal growth between roughened and polished surfaces, the two denture materials, and nicotine-treated and control groups. In the case of the Ivocap polished discs, the results of dry weight analysis and MTT analysis were contradictory. While the fungal viability data revealed higher fungal colonization on nicotine-treated polished Ivocap discs, they did not significantly differ from the control, whereas the values from the nicotine-treated group did significantly increase when compared to the control in the dry weight analysis. These results may be due to the variation in the assays employed. MTT assay measured viable cells where as the dead cells that were taken into consideration in the dry weight assay. The minimum inhibitory concentration of nystatin in the planktonic baseline group without denture disc and nicotine treatment was $1.56 \mu \mathrm{g} / \mathrm{ml}$, which was in agreement with previous studies by Anil and Ellepola When Ivocap denture discs were introduced, the nystatin MIC increased to $12.5 \mu \mathrm{g} / \mathrm{ml}$, three times more than baseline reading. The biofilm grown on Lucitone 199 denture discs, the nystatin MIC increased to $25 \mu \mathrm{g} / \mathrm{ml}$, four times more than baseline reading. When nicotine and denture discs were added, the nystatin MIC further increased to $50 \mu \mathrm{g} / \mathrm{ml}$, five times more than the baseline reading. While surface topography did not affect the efficacy of nystatin treatment, the denture material and tobacco treatment raised the MIC three to five folds as compared to the baseline group. This may be the first study that tested biofilm-grown cells' susceptibility to the anti-fungal agent nystatin. Further studies are needed to investigate the surface topography quantitatively, the hardness and density of the denture materials, other denture materials, other antifungal agents, and the effects of different concentrations of nicotine and cotinine.

\section{Conclusion}

This study assessed the influence of surface topography, denture material, and tobacco component treatment on C. albicans biofilm growth. The data obtained from this study indirectly suggest that the factors examined in this study may play an influential role in fungalinduced denture stomatitis. The study also demonstrated significant differences in fungal growth on polished and roughened surfaces, and significant differences in fungal growth on different types of acrylic discs, with a higher degree of biofilm formed on Lucitone 199 surfaces. Furthermore, tobacco components appeared to enhance biofilm formation, and biofilm fungal cells were more resistant to nystatin than the planktonic cells. Within the limitations of this study, it suggests that when adjustments are made to dentures, they should be polished prior to placement in an attempt to reduce the degree of fungal growth. In immune compromised patients, denture topography and smoking habits along with increased resistance to anti-fungal agents may increase the risk of fungal induced denture-stomatitis. Therefore, the antifungal treatment in these patients may have to be more closely monitored.

\section{Acknowledgments}

The study was partially funded by grant from American Prosthodontic Society and College of Dentistry Alumni award.

\section{Conflicts of interest}

The authors have no conflict of interest with the study or the manufacturers of the materials employed in the study.

\section{References}

1. Budtz-Jorgensen E. Clinical aspects of Candida infection in denture wearers. J Am Dent Assoc. 1978;96(3):474-479.

2. Newton A. Denture sore mouth: a possible aetiology. $\mathrm{Br}$ Dent $\mathrm{J}$. 1962;112:357-360

3. Wilson J. The aetiology, diagnosis and management of denture stomatitis Br Dent J. 1998;185(8):380-384.

4. Gendreau L, Loewy ZG. Epidemiology and etiology of denture stomatitis. J Prosthodont. 2011;20(4):251-260.

5. Budtz-Jorgensen E. The significance of Candida albicans in denture stomatitis. Scand J Dent Res. 1974;82(2):151-190.

6. Kovac-Kavcic M, Skaleric U. The prevalence of oral mucosa lesions in a population in Ljubljana, Slovenia. J Oral Pathol Med. 2000;29(7):331-335.

7. Coco BJ, Bagg J, Cross LJ, et al. Mixed Candida albicans and Candida glabrata populations associated with the pathogenesis of denture stomatitis. Oral Microbiol Immunol. 2008;23(5):377-383.

8. Emami E, Seguin J, Rompre PH, et al. The relationship of myceliated colonies of Candida albicans with denture stomatitis: an in vivo/in vitro study. Int J Prosthodont. 2007;20(5):514-520.

9. Nevalainen MJ, Narhi TO, Ainamo A. Oral mucosal lesions and oral hygiene habits in the home-living elderly. J Oral Rehabil. 1997;24(5):332-337.

10. Mikkonen M, Nyyssonen V, Paunio I, et al. Oral hygiene, dental visits and age of denture for prevalence of denture stomatitis. Community Dent Oral Epidemiol. 1984;12(6):402-405.

11. Khasawneh S, al-Wahadni A. Control of denture plaque and mucosal inflammation in denture wearers. J Ir Dent Assoc. 2002;48(4):132-138. 
12. Cannon RD, Holmes AR, Mason AB, et al. Oral Candida: clearance, colonization, or candidiasis? J Dent Res. 1995;74(5):1152-1161.

13. Campos MS, Marchini L, Bernardes LA, et al. Biofilm microbial communities of denture stomatitis. Oral Microbiol Immunol. 2008;23(5):419-424.

14. Verran J, Maryan CJ. Retention of Candida albicans on acrylic resin and silicone of different surface topography. $J$ Prosthet Dent. 1997;77(5):535-539.

15. Abu-Elteen KH, Abu-Alteen RM. The prevalence of Candida albicans populations in the mouths of complete denture wearers. New Microbiol. 1998;21(1):41-48.

16. Dagistan S, Aktas AE, Caglayan F, et al. Differential diagnosis of dentureinduced stomatitis, Candida, and their variations in patients using complete denture: a clinical and mycological study. Mycoses. 2009;52(3):266-271.

17. Davenport JC. The oral distribution of candida in denture stomatitis. $\mathrm{Br}$ Dent J. 1970;129(4):151-156.

18. Quirynen M, Bollen CM. The influence of surface roughness and surfacefree energy on supra- and subgingival plaque formation in man. A review of the literature. J Clin Periodontol. 1995;22(1):1-14.

19. King RD, Lee JC, Morris AL. Adherence of Candida albicans and other Candida species to mucosal epithelial cells. Infect Immun. 1980;27(2):667-674.

20. Rueggeberg FA. From vulcanite to vinyl, a history of resins in restorative dentistry. J Prosthet Dent. 2002;87(4):364-379.

21. Renu Tandon SG, Samarth Kumar Agarwal. Denture base materials: From past to future. Indian Journal of Dental Sciences. 2010;2:33-39.

22. Vivadent I. Ivocap the Successful Technique Heat Curing Injection System. 2013.

23. Barbeau J, Seguin J, Goulet JP, et al. Reassessing the presence of Candida albicans in denture-related stomatitis. Oral Surg Oral Med Oral Pathol Oral Radiol Endod. 2003;95(1):51-59.

24. Arendorf TM, Walker DM, Kingdom RJ, et al. Tobacco smoking and denture wearing in oral candidal leukoplakia. $\mathrm{Br}$ Dent $J$. 1983;155(10):340-343
25. Hatsukami DK, Hecht SS, Hennrikus DJ, et al. Biomarkers of tobacco exposure or harm: application to clinical and epidemiological studies. Nicotine Tob Res. 2003;5(3):387-396.

26. Ciolino LA, McCauley HA, Fraser DB, et al. The relative buffering capacities of saliva and moist snuff: implications for nicotine absorption. J Anal Toxicol. 2001;25(1):15-25.

27. Beckett AH, Gorrod JW, Jenner P. A possible relation between pKa 1 and lipid solubility and the amounts excreted in urine of some tobacco alkaloids given to man. J Pharm Pharmacol. 1972;24(2):115-120.

28. Cote F, Letourneau C, Mullard G, et al. Estimation of nicotine and tar yields from human-smoked cigarettes before and after the implementation of the cigarette ignition propensity regulations in Canada. Regul Toxicol Pharmacol. 2011;61(3 Suppl):S51-S59.

29. Robson N, Bond A, Wolff K. Salivary nicotine and cotinine concentrations in unstimulated and stimulated saliva. African Journal of Pharmacy and Pharmacology. 2010;4(2):61-65.

30. Hazen EL, Brown R. Two antifungal agents produced by a soil actinomycete. Science. 1950;112(2911):423.

31. Stewart GT. Laboratory and Clinical Studies with Nystatin in Postantibiotic Mycotic Infections. Br Med J. 1956;1:658-660.

32. Ellepola AN, Samaranayake LP. Oral candidal infections and antimycotics. Crit Rev Oral Biol Med. 2000;11(2):172-198.

33. Schafer-Korting M, Blechschmidt J, Korting HC. Clinical use of oral nystatin in the prevention of systemic candidosis in patients at particular risk. Mycoses. 1996;39(9-10):329-339.

34. Ellepola AN, Samaranayake LP. Adhesion of oral Candida albicans isolates to denture acrylic following limited exposure to antifungal agents. Arch Oral Biol. 1998;43(12):999-1007.

35. McCourtie J, MacFarlane TW, Samaranayake LP. A comparison of the effects of chlorhexidine gluconate, amphotericin B and nystatin on the adherence of Candida species to denture acrylic. J Antimicrob Chemother. 1986;17(5):575-583. 